\title{
Galactic structure from trigonometric parallaxes of star-forming regions
}

\author{
Mark J. Reid \\ Harvard-Smithsonian Center for Astrophysics, Cambridge, MA, USA \\ email: reid@cfa.harvard.edu
}

\begin{abstract}
Recently, astrometric accuracy approaching $\sim 10 \mu$ as has become routinely possible with Very Long Baseline Interferometry. Since, unlike at optical wavelengths, interstellar dust is transparent at radio wavelengths, parallaxes and proper motions can now be measured for massive young stars (with maser emission) across the Galaxy, enabling direct measurements of the spiral structure of the Milky Way. Fitting the full 3D position and velocity vectors to a simple model of the Galaxy yields extremely accurate values for its fundamental parameters, including the distance to the Galactic Center, $\mathrm{R}_{0}=8.38 \pm 0.18 \mathrm{kpc}$, and circular rotation at the Solar Circle, $\Theta_{0}=243 \pm 7 \mathrm{~km} \mathrm{~s}^{-1}$. The rotation curve of the Milky Way, based for the first time on 'gold standard' distances and complete 3D information, appears to be very flat.
\end{abstract}

Keywords. Galaxy: fundamental parameters, Galaxy: structure, Galaxy: disk, HiI regions, stars: formation

\section{Introduction}

Surprisingly, the nature of the spiral structure of the Milky Way remains largely unknown after nearly a century of intense observational studies. The primary reasons are that dust obscures most of the Galaxy's disk from view optically and distances are exceedingly great and difficult to measure. The discovery of the $21 \mathrm{~cm}$ spectral line of atomic hydrogen, and later millimeter-wave lines of molecular carbon monoxide (CO) gas, gave hope that one could map the Milky Way, because these radio lines were not affected by interstellar dust and their observed velocities could be used to estimate (kinematic) distances. However, kinematic distances are plagued by both ambiguities for sources observed inside the 'Solar Circle' and large uncertainties from unknown non-circular motions.

Recently, astrometric techniques using Very Long Baseline Interferometry (VLBI) have improved dramatically. Currently one can achieve single-epoch relative position uncertainties approaching $\sim 10 \mu$ as for sources separated by $\sim 1^{\circ}$ on the sky. This allows one to measure absolute parallaxes (relative to background quasars) to bright, compact radio sources throughout the Galaxy with accuracies comparable to the target accuracy of the Gaia mission. This paper discusses recent parallax results for maser emission associated with the formation of massive and extremely young stars with the NRAO $†$ Very Long Baseline Array (VLBA), the Japanese VERA project, and the European VLBI Network $(E V N)$, and their impact on our understanding of Galactic structure.

$\dagger$ The National Radio Astronomy Observatory is a facility of the US National Science Foundation operated under cooperative agreement by Associated Universities, Inc. 


\section{Example Parallax Results}

Numerous VLBI parallaxes can now be found in the literature (e.g., Xu et al. 2006; Honma et al. 2007; Menten et al. 2007; Rygl et al. 2010). Here we show two examples of data that demonstrate the potential of astrometric VLBI to map the Milky Way.

The first example is Sgr B2, a massive star-forming region located near the Galactic Center. Reid et al. (2009a) reported a VLBA parallax of $0.129 \pm 0.012$ mas (see Fig. 1), corresponding to a distance of $7.8 \pm 0.8 \mathrm{kpc}$, based on $\mathrm{H}_{2} \mathrm{O}$ masers in Sgr B2N and Sgr B2M. These star-forming regions are probably $\approx 0.1 \mathrm{kpc}$ nearer than Sgr $\mathrm{A}^{*}$, which defines the Galactic Center, since (i) Sgr B2's projected distance is only $\approx 0.09 \mathrm{kpc}$ from Sgr $A^{*}$ and (ii) its $3 \mathrm{D}$ velocity (using its measured proper motion) suggests a line-ofsight offset of $0.130 \pm 0.06 \mathrm{pc}$ (assuming a low-eccentricity Galactic orbit) toward the Sun. Adjusting the distance for this $0.1 \mathrm{kpc}$ offset from the Galactic Center gives a direct measurement of $R_{0}=7.9 \pm 0.8 \mathrm{kpc}$. Ongoing and future observations may yield a more accurate results.
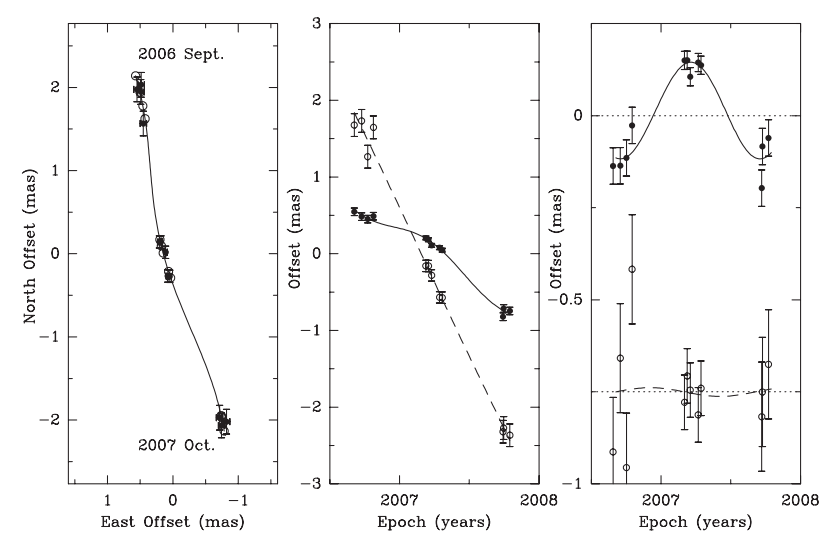

Figure 1. Parallax to the Galactic Center $\mathrm{H}_{2} \mathrm{O}$ maser source Sgr B2 from Reid et al. (2009a), yielding $\mathrm{R}_{0}=7.9 \pm 0.8 \mathrm{kpc}$. (left) Positions on the sky of one maser spot measured relative to three background sources. (middle) Eastward (filled circles, solid line) and northward (open circles, dashed line) offsets versus time. (right) Same as middle panel, but with proper motion removed, showing only the parallax signature.

A second example is the $\mathrm{H}_{2} \mathrm{O}$ maser source $\mathrm{W} 49 \mathrm{~N}$, which may be the most luminous maser in the Galaxy. A preliminary VLBA parallax to this source (see Fig. 2) places it at a distance of $12.2 \pm 0.9 \mathrm{kpc}$. By comparing its Galactic longitude $\left(43^{\circ}\right)$ and local-standardof-rest (LSR) velocity ( $\approx 8 \mathrm{~km} \mathrm{~s}^{-1}$ ) with molecular clouds in CO longitude--velocity plots, we know that this star-forming region is located in the Perseus spiral arm. This locates the Perseus spiral arm in the first quadrant of the Galaxy to better than $10 \%$ accuracy - a key result for mapping spiral structure.

\section{Preliminary Results on Spiral Structure}

There are now more than 90 parallax measurements in total for massive star-forming regions measured by the VLBA, VERA, and EVN. Fig. 3 shows the locations of the star-forming regions, superposed on an artist's conception of the Milky Way. The two sources discussed in the previous section (Sgr B2 and W49N) are labeled in the figure. The sources are coded by spiral arm, obtained by matching Galactic longitudes and LSR 


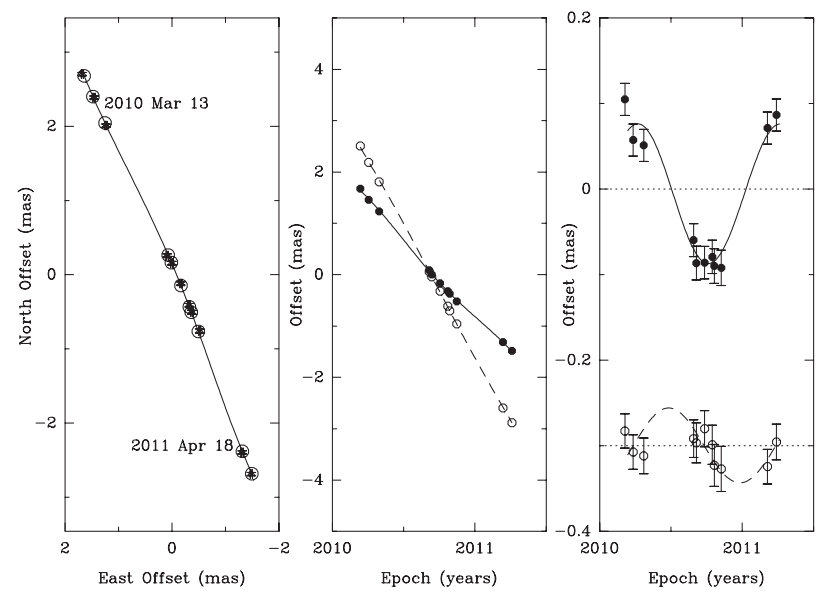

Figure 2. Preliminary parallax to the distant $\mathrm{H}_{2} \mathrm{O}$ maser source W49N, yielding $\mathrm{R}_{0}=12.2 \pm 0.9 \mathrm{kpc}$ by B. Zhang et al. (in prep.). (left) Positions on the sky of one maser spot measured relative to three background sources. (middle) Eastward (solid line) and northward (dashed line) offsets versus time. (right) Same as middle panel, but with proper motion removed, showing only the parallax signature. This result for the first time accurately locates the Perseus spiral arm of the Milky Way toward Galactic longitude $43^{\circ}$.

velocities with atomic hydrogen $(\mathrm{H})$ and molecular $\mathrm{CO}$ emission patterns in longitudevelocity plots. We sare now starting to precisely locate the major spiral arms of the Milky Way, including the Outer, Perseus, Sagittarius-Carina, and Crux-Centaurus-Scutum arms. The Local arm has many sources and must be a prominent feature of the Milky Way. The inner region of the Galaxy, strongly influenced by the bar, looks very complicated.

Fig. 4 plots the logarithm of Galactocentric radius, $R$, versus Galactocentric azimuth, $\beta$. Log-periodic spirals given by $\log _{10}\left(R / R_{\text {ref }}\right)=-\left(\beta-\beta_{\text {ref }}\right) \tan \psi$, where $R_{\text {ref }}$ and $\beta_{\text {ref }}$ are the radius and azimuth of the start of the arm and $\psi$ is the arm's pitch angle, map to straight lines on such a plot. Sections of spiral arms with Galactocentric radius greater than $\sim 8 \mathrm{kpc}$ (including the Outer, Perseus, and Local arms) appear to have pitch angles near $13^{\circ}$. Sections of spiral arms inside of $\sim 8 \mathrm{kpc}$ may have flatter pitch angles, although more data will be needed to confirm this preliminary indication.

\section{Fundamental Parameters of the Milky Way}

The astrometric VLBI observations yield accurate proper motions as well as parallaxes. Since the line-of-sight (i.e., LSR) velocities are also available, one has full 3D position and velocity information for each star-forming region. The combined data set for over 90 sources can be modeled to estimate the fundamental parameters of the Galaxy: $R_{0}$, the distance of the Sun from the Galactic Center, and $\Theta_{0}$, the circular rotation speed at the Solar Circle.

Following Reid et al. (2009b), we take the source coordinates as independent variables and measurements of parallax, proper motion in right ascension and declination, and LSR velocity as data. Data uncertainties are the sums in quadrature of measurement errors and the effects of 'virial noise,' estimated to be $7 \mathrm{~km} \mathrm{~s}^{-1}$ per coordinate. We then model the Galaxy with axially symmetric rotation, specified by $\mathrm{R}_{0}, \Theta_{0}$, and $\partial \Theta / \partial R$ (to allow for a sloped rotation curve). In addition, we allow for an average systematic motion of the star-forming regions toward the Galactic Center, $\left\langle U_{\mathrm{s}}\right\rangle$ and in the direction 


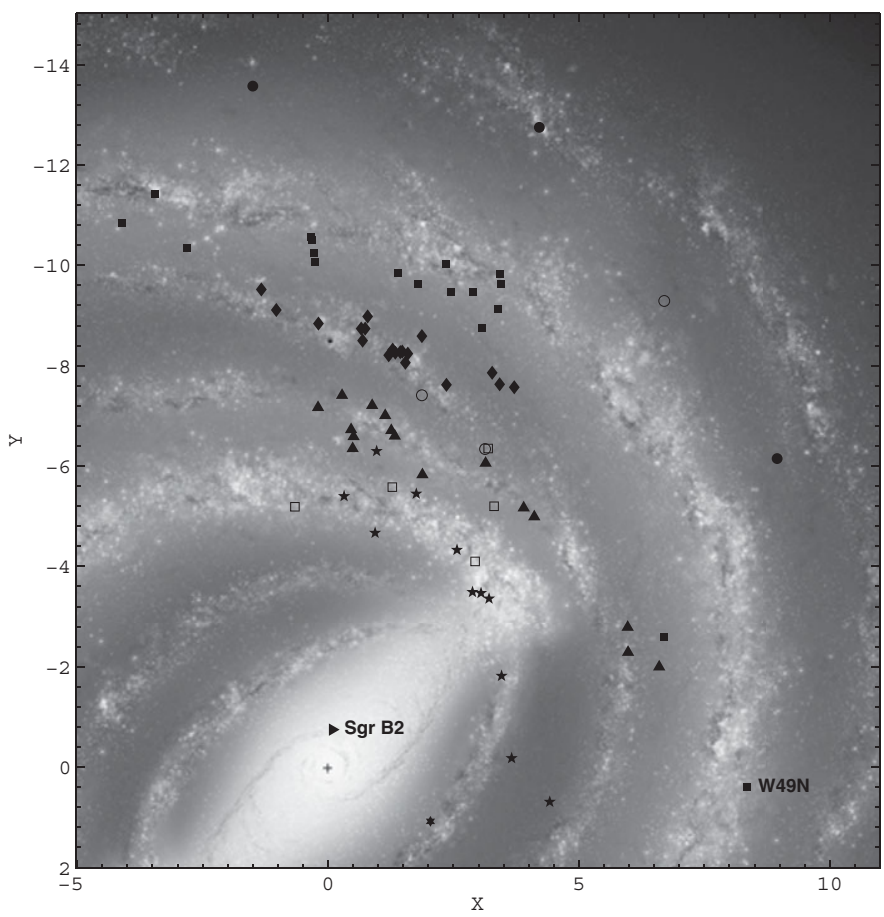

Figure 3. Locations of massive star-forming regions from trigonometric parallaxes measured by the VLBA, the Japanese VERA project, and the EVN. These are superposed on an artist's conception of the Milky Way by Robert Hurt.

of Galactic rotation, $\left\langle V_{\mathrm{s}}\right\rangle$. We adopt the non-circular component of the Sun's orbit (the Solar Motion) from Schönrich et al. (2010).

We expect that the kinematics of some star-forming regions will not be well fitted by our very simple model. For example, sources near the Galactic bar or those associated with 'superbubbles,' are expected to have significant non-circular motions. To avoid potentially subjective editing of data, we fit all data using an outlier-tolerant Bayesian approach (Markov-chain Monte Carlo trials accepted according to the Metropolis-Hastings algorithm) described by Sivia \& Skilling (2006), called 'a conservative formulation.'

Using this approach, we find best estimates of $\mathrm{R}_{0}=8.38 \pm 0.18 \mathrm{kpc}, \Theta_{0}=243 \pm 7 \mathrm{~km}$ $\mathrm{s}^{-1}$, and $\partial \Theta / \partial R=-0.4 \pm 0.7 \mathrm{~km} \mathrm{~s}^{-1} \mathrm{kpc}^{-1}$. We also find average source peculiar-motion components of $\left\langle U_{\mathrm{s}}\right\rangle=6 \pm 2 \mathrm{~km} \mathrm{~s}^{-1}$ and $\left\langle V_{\mathrm{s}}\right\rangle=-8 \pm 2 \mathrm{~km} \mathrm{~s}^{-1}$. These results are similar to, but more accurate than, those of Reid et al. (2009b), which were based on only 18 sources and assumed earlier values of the Solar Motion by Dehnen \& Binney (1998). (Note that the values of $\Theta_{0}$ and $\left\langle V_{\mathrm{s}}\right\rangle$ are sensitive to the value of the component of Solar Motion in the direction of Galactic rotation.)

\section{Rotation Curve}

Measurement of Doppler shift and proper motion are obtained in a heliocentric, not a Galactocentric, frame. Therefore, to construct a (Galactocentric) rotation curve for the Milky Way requires adding the full orbital velocity vector of the Sun to the observed source vectors. Almost all rotation curves constructed in the past have been based only on 1D (Doppler) velocities and an assumed value for $\Theta_{0}$. Essentially, what one assumes for $\Theta_{0}$ determines the scale of the rotation curve. 


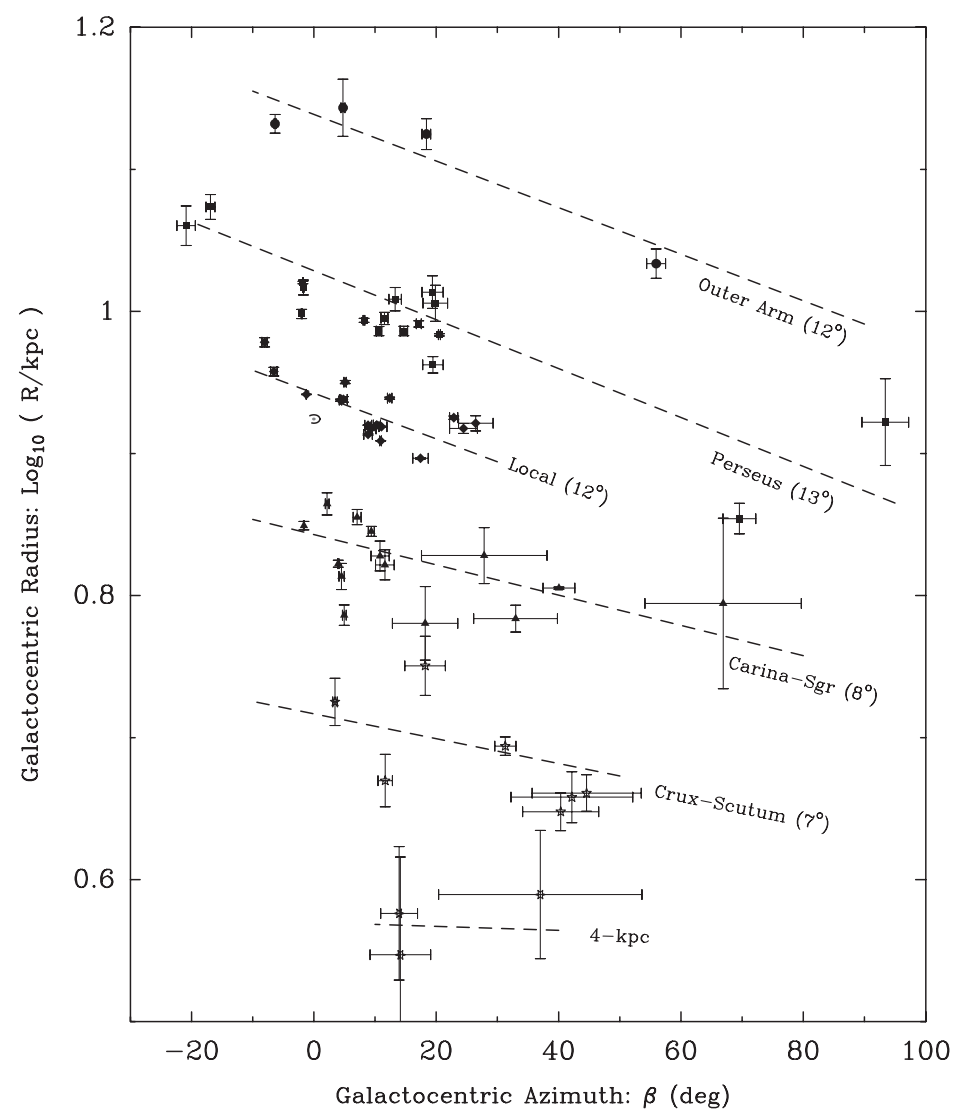

Figure 4. Spiral-arm pitch-angle plot of the logarithm of Galactocentric radius versus Galactocentric azimuth. Log-periodic spirals map to straight lines on such a plot. Sources are coded by different symbol shapes to indicate spiral-arm designation, which was obtained by matching Galactic longitudes and LSR velocities with atomic $\mathrm{H}$ and molecular $\mathrm{CO}$ emission patterns in longitude-velocity plots.

From parallaxes and proper motions of a large number of high-mass star-forming regions, we now have full 3D locations and velocity vectors. By modeling the entire data set, we can now independently solve for $\Theta_{0}$ (as described in Sect. 4), and then generate the projection of each source's $3 \mathrm{D}$ velocity vector in the direction of Galactic rotation to obtain a rotation curve. Fig. 4 shows this rotation curve. Note, with our 'gold standard' distances and $3 \mathrm{D}$ motions, we now can reliably conclude that the rotation speed of the Galaxy is nearly flat at $\approx 243 \mathrm{~km} \mathrm{~s}^{-1}$ between Galactocentric radii of 4 and $14 \mathrm{kpc}$.

\section{Conclusions}

Very Long Baseline Interferometry is now being used to measure parallaxes and proper motions to regions of high-mass star formation. Single-epoch position accuracies approaching $\sim 10 \mu$ as have been achieved, leading to parallaxes for sources across the Milky Way. Results from the VLBA, VERA, and EVN are making it possible to reliably map the spiral structure of the Milky Way for the first time. Preliminary results show that the Milky Way has four major, gas-rich spiral arms, and some minor arms in its inner region near the bar. Pitch angles are near $13^{\circ}$ for the outer sections of spiral arms, and may be smaller for the inner sections. 


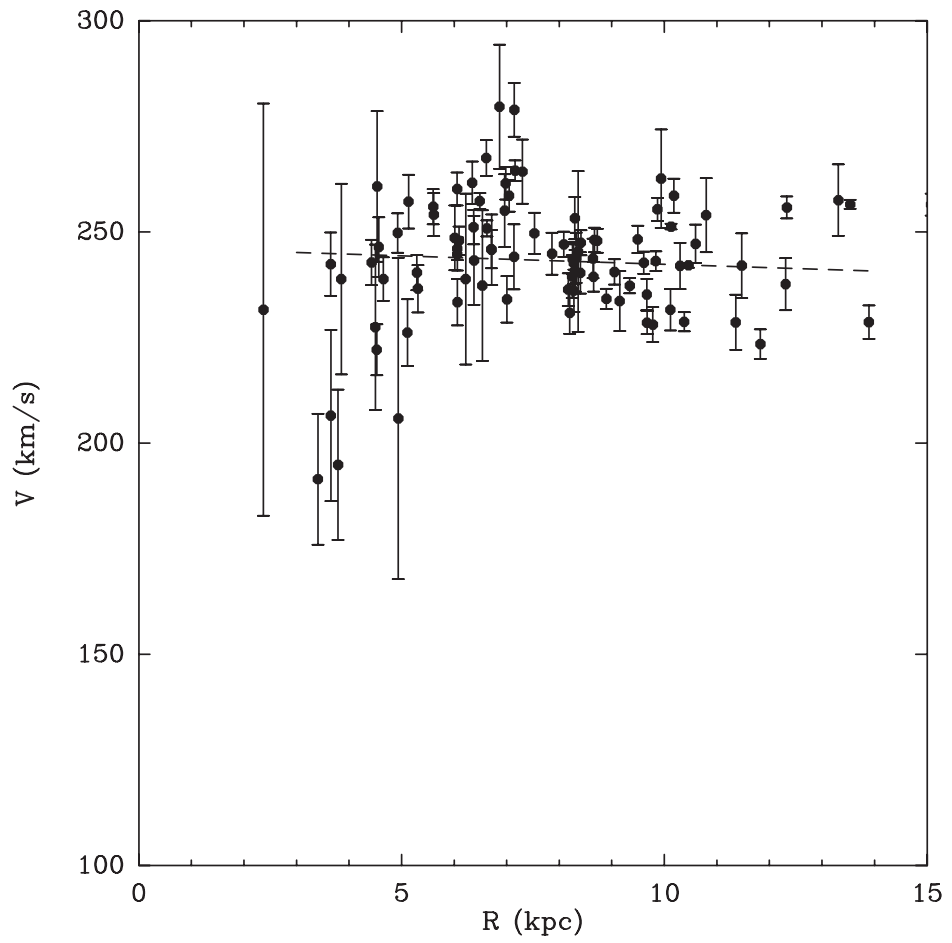

Figure 5. Rotation curve based on parallaxes and proper motions of high-mass star-forming regions. This rotation curve, for the first time, uses 'gold standard' distances and full 3D motions. The dashed line indicates the slope in the rotation curve from fits to all data described in Sect. 4 .

As originally pointed out by Reid et al. (2009b), and now confirmed with large numbers of new observations, star-forming regions 'counter rotate' by $\approx 8 \mathrm{~km} \mathrm{~s}^{-1}$, when adjusted for the new Solar Motion of Schönrich et al. (2010). This suggests that massive starforming regions might be formed near apocenter in slightly elliptical orbits about the Galactic Center.

Fitting the full 3D position and velocity measurements with a simple axially symmetric model for the Milky Way's rotation, yields $R_{0}=8.38 \pm 0.18 \mathrm{kpc}, \Theta_{0}=243 \pm 7 \mathrm{~km} \mathrm{~s}{ }^{-1}$, and a nearly flat rotation curve.

\section{References}

Dehnen, W. \& Binney, J. J. 1998, MNRAS, 298, 387

Honma, M., Bushimata, T., Choi, Y. K., et al. 2007, PASJ, 59, 889

Menten, K. M., Reid, M. J., Forbrich, J., \& Brunthaler, A. 2007, A\&AA, 474, 515

Reid, M. J., Menten, K. M., Zheng, X. W., et al. 2009, ApJ, 700, 137

Reid, M. J., Menten, K. M., Zheng, X. W., Brunthaler, A., \& Xu, Y. 2009, ApJ, 705, 1548

Rygl, K. L. J., Brunthaler, A., Reid, M. J., Menten, K. M., van Langevelde, H. J., \& Xu, Y. 2010, A\&A, 511, 2

Schönrich, R., Binney, J., \& Dehnen, W. 2010, MNRAS, 403, 1829

Sivia, D. \& Skilling, J. 2006, in: Data Analysis: A Bayesian Tutorial, $2^{\text {nd }}$ ed. (Oxford Univ. Press), p. 168

Xu, Y., Reid, M. J., Zheng, X. W., \& Menten, K. M. 2006, Science, 311, 54 